\title{
Solveurs multigrilles et méthode asymptotique numérique
}

\author{
Anas Mtalaa* - Jean-Marc Cadou** ${ }^{* *}$ Michel Potier-Ferry* \\ * Laboratoire de Physique et Mécanique des Matériaux \\ I.S.G.M.P. - Ile du Saulcy \\ Université Paul Verlaine \\ F-57045 Metz \\ anas.mtalaa@univ-metz.fr \\ ** Laboratoire Génie Mécanique et Matériaux \\ Rue de Saint Maudé, BP 92116 \\ F-56321 Lorient cedex \\ jean-marc.cadou@univ-ubs.fr
}

\begin{abstract}
RÉSUMÉ. L'objectif de ce travail est la mise au point de techniques numériques pour la résolution de problèmes non linéaires à très grand nombre de degrés de liberté. Nous proposons de coupler des techniques multigrilles à la méthode asymptotique numérique (MAN) qui associe une méthode de perturbation à la méthode des éléments finis.

ABSTRACT. The aim of this work is to build up new numerical techniques to solve non-linear large scale problems. We propose to couple multigrid techniques with the asymptotic numerical method (ANM) which associates a perturbation technique with the finite element method.

MOTS-CLÉS : méthode asymptotique numérique, perturbation, problèmes à grand nombre d'inconnues, solveurs multigrilles.

KEYWORDS: asymptotic numerical method, perturbation, large scale problems, solver multigrid.
\end{abstract}

DOI:10.3166/REMN.16.813-826 @ 2007 Lavoisier, Paris 


\section{Introduction}

Pour la résolution des problèmes à grand nombre de degrés de liberté (de plusieurs centaines de milliers à quelques millions) les méthodes itératives sont largement utilisées car elles permettent de réduire le nombre de réels à stocker. Par ailleurs, l'utilisation de ces méthodes est parfois indispensable car elles permettent souvent un gain significatif en temps de calcul par rapport aux méthodes directes. Pour résoudre ces problèmes non linéaires à grand nombre de degrés de liberté ( $\mathrm{ddl})$, nous proposons de combiner la méthode asymptotique numérique, MAN (Potier-Ferry et al., 2004), avec une méthode de résolution itérative appelée méthode multigrille. Cette méthode de résolution permet d'éviter de triangulariser des matrices de grande taille. La méthode multigrille retenue dans ce travail est une méthode à deux grilles.

L'utilisation d'une méthode asymptotique numérique conduit à transformer le problème non linéaire initial en une succession de problèmes linéaires ayant tous le même opérateur tangent et se différenciant par le vecteur second membre. Cette méthode a été utilisée avec succès pour résoudre des problèmes de vibrations non linéaires (Azrar et al., 2002), de contact unilatéral (Elhage-Hussein et al., 1998), de mécanique des fluides (Cadou et al., 2001), de viscoplasticité (Descamps et al., 1997), de plasticité (Braikat et al., 1997) ainsi que des benchmarks de mise en forme (Abichou et al., 2002). La discrétisation de ces problèmes linéaires est classiquement effectuée à l'aide de la méthode des éléments finis ce qui conduit au système linéaire discret :

$$
\left[k_{t}\left(u_{0}\right)\right]\left\{u_{i}\right\}=f_{i}
$$

où $k_{t}\left(u_{0}\right)$ désigne la matrice tangente calculée au point solution $u_{0}, f_{i}$ désigne le second membre et $u_{i}$ est l'inconnue. L'indice $i$ désigne l'ordre de la méthode de la perturbation et son maximum est généralement choisi entre 15 et 20 . La même matrice tangente est utilisée lors de la résolution de chaque problème linéaire [1]. Les seconds membres $f_{i}$ dépendent des solutions aux ordres précédents. Dans le cas des problèmes de dimension moyenne (inférieure à $20000 \mathrm{ddl}$ ), la résolution des problèmes linéaires [1] est réalisée par une méthode directe. La matrice tangente est ainsi triangulée une seule fois, à l'ordre un, et des montées-descentes sont réalisées pour déterminer les solutions $u_{i}$ aux ordres supérieurs.

Pour la résolution des problèmes à grand nombre d'inconnues, nous proposons dans cette étude l'utilisation d'une méthode itérative pour résoudre les 15-20 problèmes linéaires [1]. Dans le cas du couplage MAN et solveur itétarif (le gradient conjugué préconditionné par exemple), des techniques numériques (Farhat et $a l ., 1994)$ pour des problèmes linéaires à matrice identique et à seconds membres différents ont été étudiées : les directions de descente du problème $i$ étaient utilisées pour le problème à l'ordre $i+1$. Néanmoins, si ce type de méthode est bien adapté pour de petits problèmes comme les problèmes d'interface issus d'une sous-structuration (Rey, 1996), elles sont trop coûteuses dans le cas de problèmes de grande dimension. Pour ces raisons, nous avons choisi d'utiliser dans ce travail, une méthode multigrille classique (Désidéri, 1998) avec 2 grilles. Dans un travail récent (El Mokhtari, 2002), il a été montré que pour des problèmes $2 \mathrm{D}$, l'utilisation d'une méthode multigrille 
pour résoudre les problèmes linéaires issus de la MAN était une alternative prometteuse. Notre objectif dans ce travail consiste à adapter le couplage de la MAN avec une méthode multigrille pour la résolution de problèmes de coques minces.

Dans ce papier, nous présentons dans un premier temps les performances et les propriétés de la méthode à 2 grilles utilisée. Dans une deuxième partie, nous présentons les résultats numériques obtenus lors de la résolution des problèmes linéaires [1]. Dans une dernière partie, nous présentons les difficultés liées à l'utilisation de cette méthode de résolution dans une stratégie pas-à-pas.

\section{Méthodes multigrilles}

Dans les sections qui suivent, le principe des méthodes multigrilles est brièvement rappellé. Pour de plus amples informations concernant les méthodes multigrilles nous renvoyons le lecteur aux références (Désidéri, 1998 ; Fish et al., 1996 ; Brandt, 1982) ainsi qu'au site internet www.mgnet.ord où une bibliographie conséquente est disponible. Dans cette étude, les caractéristiques et les propriétés de ces méthodes sont décrites sur des exemples numériques.

\subsection{Description de la méthode à 2 grilles}

La méthode à 2 grilles utilise deux niveaux de maillage, une grille grossière et une grille fine. Dans nos applications numériques, la grille fine est obtenue en affinant le maillage de la grille grossière, figure 1. Le passage d'informations entre les deux grilles s'effectue à l'aide d'opérateurs de prolongement $\mathrm{P}$ (de la grille fine à la grille grossière) et de restriction $\mathrm{R}$ (de la grille grossière à la grille fine). Pour définir ces opérateurs de passage entre grille fine $\left(\Omega_{h}\right)$ et grille grossière $\left(\Omega_{2 h}\right)$ considérons les deux niveaux de maillage de la figure 1. En notant les informations de la grille fine $q_{h}$

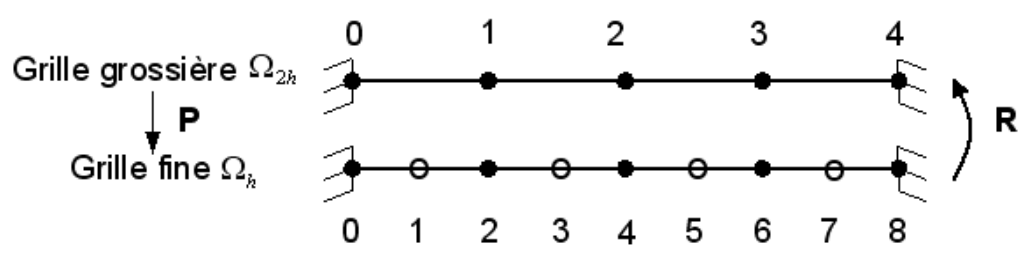

Figure 1. Exemple de grilles dans le cas monodimensionnel

(par exemple le déplacement aux nœuds) et celles de la grille grossière $q_{2 h}$, on peut écrire à l'aide des opérateurs $\mathrm{P}$ et $\mathrm{R}$ les relations suivantes :

$$
q_{h}=P . q_{2 h}, q_{2 h}=R \cdot q_{h}
$$


A titre d'illustration, considérons le cas de maillages 1D imbriqués proposé sur la figure 1. Une interpolation linéaire est utilisée pour le passage de la grille grossière vers la grille fine :

$$
q_{2 h}=\left\{\begin{array}{c}
q_{1} \\
q_{2} \\
q_{3}
\end{array}\right\} \longrightarrow q_{h}=\left\{\begin{array}{c}
q_{1}^{\prime}=\left(q_{0}+q_{1}\right) / 2 \\
q_{2}^{\prime}=q_{1} \\
q_{3}^{\prime}=\left(q_{1}+q_{2}\right) / 2 \\
q_{4}^{\prime}=q_{2} \\
q_{5}^{\prime}=\left(q_{2}+q_{3}\right) / 2 \\
q_{6}^{\prime}=q_{3} \\
q_{7}^{\prime}=\left(q_{3}+q_{4}\right) / 2
\end{array}\right\}
$$

L'opérateur de prolongement $P$ est alors défini par :

$$
P=\left[\begin{array}{ccc}
1 / 2 & 0 & 0 \\
1 & 0 & 0 \\
1 / 2 & 1 / 2 & 0 \\
0 & 1 & 0 \\
0 & 1 / 2 & 1 / 2 \\
0 & 0 & 1 \\
0 & 0 & 1 / 2
\end{array}\right]
$$

A partir de la figure 1 on peut également définir l'opérateur de restriction $R$ :

$$
R=\left[\begin{array}{lllllll}
0 & 1 & 0 & 0 & 0 & 0 & 0 \\
0 & 0 & 0 & 1 & 0 & 0 & 0 \\
0 & 0 & 0 & 0 & 0 & 1 & 0
\end{array}\right]
$$

Dans le cadre de notre travail sur le flambage non linéaire des coques minces, les éléments finis utilisés sont des triangles et plus précisément des DKT (Batoz et al., 1990). Les grilles fines sont alors déduites des grilles grossières (voir figure 2). Les opérateurs de prolongement et de restriction sont obtenus de la même manière que dans le cas $1 \mathrm{D}$ de la figure 1.

\subsection{Description de l'algorithme de résolution}

L'algorithme de résolution de la méthode à deux grilles est le suivant :

1. lissage (2 ou 3 itérations d'un solveur itératif) sur la grille fine,

2. calcul du défaut (résidu) sur la grille fine,

3. restriction du résidu sur la grille grossière,

4. résolution du système sur la grille grossière,

5. prolongement de l'équation sur la grille fine,

6. si le résidu sur la grille fine est trop grand (ou du moins supérieur à une précision demandée), on recommence un autre cycle. 


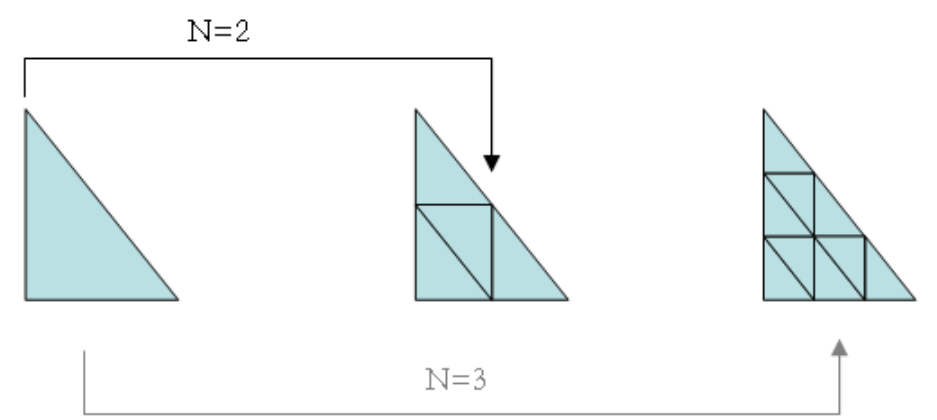

Figure 2. Exemple de grilles dans le cas bidimensionnel ( $N$ : Nombre de subdivisions)

L'étape 1 (le lissage) correspond à 2 ou 3 itérations d'un solveur itératif. Dans cette étude nous utilisons le gradient conjugué avec un préconditionneur de type factorisation incomplète de Crout (Joly et al., 1994). Ce dernier est un préconditionneur à niveau. La matrice factorisée est ainsi enrichie lorsque le niveau augmente. Par défaut nous utiliserons le niveau 0 car ce dernier associe rapidité de convergence et une matrice de préconditionnement peu coûteuse en temps de calcul et en place mémoire. Plusieurs solveurs itératifs ont été testés durant cette étape de lissage tels que les méthodes de Jacobi et de Gauss-Seydel. Pour les problèmes considérés dans ce travail, une meilleure convergence de la méthode à deux grilles a été obtenue avec un gradient conjugué préconditionné. Pour l'étape 4, à savoir la résolution sur la grille grossière, nous utilisons une méthode directe : la factorisation de Crout.

\subsection{Applications numériques de la méthode à deux grilles}

Nous présentons à présent les résultats de calculs en 3D. L'objectif est de rappeler les propriétés et les caractéristiques des méthodes multigrilles. Nous nous plaçons dans le cadre de l'élasticité linéaire. Les éléments utilisés pour mailler les structures sont des éléments DKT 18 (Discrete Kirchhoff Triangle) qui comptent 3 nœuds et 6 ddl par nœud. Ces éléments sont particulièrement adaptés dans le cas de calculs de coques minces où le cisaillement transverse est négligeable (Batoz et al., 1990).

\subsection{Influence du nombre de ddl}

Nous considérons l'exemple d'une plaque encastrée sur ses quatre côtés et soumise à une force de flexion en son centre. Nous étudions la méthode à deux grilles avec différents maillages. 


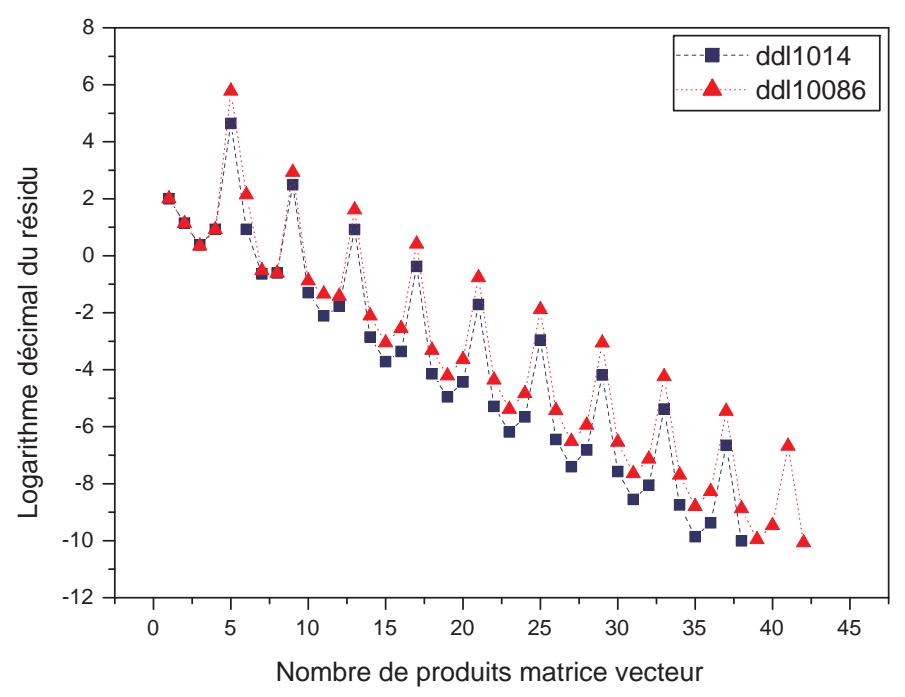

Figure 3. Comportement du résidu de la méthode à deux grilles pour deux maillages différents

\begin{tabular}{|l|c|c|c|c|c|c|}
\hline Nombre de ddl & $\begin{array}{c}\text { Grille grossière } \\
\text { Grille fine }\end{array}$ & $\begin{array}{c}1014 \\
3750\end{array}$ & $\begin{array}{c}2646 \\
10086\end{array}$ & $\begin{array}{c}3750 \\
14406\end{array}$ & $\begin{array}{c}5766 \\
22326\end{array}$ & $\begin{array}{c}10086 \\
39366\end{array}$ \\
\hline Nombre de produits matrice-vecteur & 38 & 39 & 39 & 39 & 42 \\
\hline
\end{tabular}

Tableau 1. Convergence de la méthode à deux grilles pour divers maillages. Le nombre de subdivisons est $N=2$

Le nombre de produits matrice-vecteur nécessaires pour obtenir la précision souhaitée (dans ce cas $\delta=10^{-10}$ ) et pour plusieurs maillages est donné dans le tableau 1. Ce nombre de produits matrice-vecteur est directement relié au nombre de cycles de la méthode à deux grilles. Nous représentons également sur la figure 3 , pour deux maillages, l'évolution du logarithme du résidu en fonction du nombre de produits matrice-vecteur. Ces résultats montrent que la convergence de la méthode à deux grilles est indépendante du nombre de ddl de la grille fine (pour un ratio du nombre de ddl de la grille fine sur un nombre de ddl de la grille grossière constant, $\mathrm{N}$ ). 


\begin{tabular}{|c|c|}
\hline Algorithme & Nb \\
\hline \hline $\mathrm{N}=2$, Grille Grossière, 3721 noeuds & 40 \\
\hline $\mathrm{N}=3$, Grille Grossière, 1681 noeuds & 60 \\
\hline $\mathrm{N}=4$, Grille Grossière, 961 noeuds & 84 \\
\hline $\mathrm{N}=5$, Grille Grossière, 625 noeuds & 100 \\
\hline $\mathrm{N}=6$, Grille Grossière, 441 noeuds & 164 \\
\hline $\mathrm{N}=8$, Grille Grossière, 256 noeuds & 416 \\
\hline $\mathrm{N}=10$, Grille Grossière, 169 noeuds & 1220 \\
\hline Gradient conjugué, 14641 noeuds & 715 \\
\hline
\end{tabular}

Tableau 2. Influence de $N$ sur le nombre de produits matrice vecteur (Nb)

\subsection{Influence du nombre de division $N$}

Toujours sur l'exemple de la plaque encastrée sur ses quatre côtés et soumise à une force de flexion en son centre nous faisons varier le nombre de division N. Nous indiquons dans le tableau 2 le nombre de produits matrice-vecteur nécessaire pour obtenir la qualité demandée. Pour tous ces tests, le nombre de ddl de la grille fine (environ 87 000) est fixé et nous faisons varier le paramètre $\mathrm{N}$, c'est-à-dire le nombre de ddl de la grille grossière. Le problème posé sur la grille fine est également résolu par la méthode du gradient conjugué préconditionné. Le préconditionneur utilisé est une factorisation incomplète de Crout et de niveau 0.

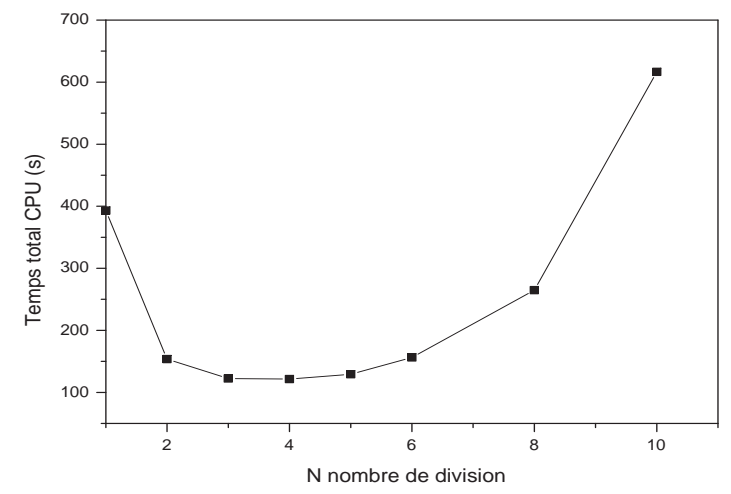

Figure 4. Evolution du temps CPU en fonction du nombre de division $N$

Il apparaît dans le tableau 2 que le nombre de produits matrice vecteur crôit avec N. Ce nombre de produits matrice vecteur est toujours inférieur à celui obtenu avec la méthode du gradient conjugué préconditionné, sauf pour une valeur de $\mathrm{N}$ supérieure à 8. Nous avons également représenté sur la figure 4 l'évolution du temps CPU en fonc- 
tion de $\mathrm{N}, \mathrm{N}=1$ correspondant à la méthode du gradient conjugué. Nous observons sur cette figure que les temps de résolution de la méthode à deux grilles sont toujours inférieurs à ceux du gradient conjugué pour des valeurs de $\mathrm{N}$ inférieurs à 8 . En effet lorsque le parmètre $\mathrm{N}$ augmente, la taille de la matrice de rigidité de la grille grossière diminue et par conséquent le temps de décomposition de cette matrice décroît. Mais à partir de $\mathrm{N}=8$ la convergence devient plus délicate, beaucoup d'itérations sont alors nécessaires pour obtenir la précision souhaitée. Le temps CPU total augmente alors en conséquence.

\section{Association méthode à deux grilles/méthode asymptotique numérique}

Nous nous intéressons dans cette section à la résolution par la méthode à deux grilles des problèmes linéaires de l'équation [1] obtenus lors de la résolution d'un problème non linéaire avec une méthode asymptotique numérique.

Les résultats numériques des sections précédentes montrent que les performances de l'algorithme à 2 grilles sont optimales pour un nombre de division proche de 2 et ceci quel que soit le nombre de ddl de la grille fine. Les applications numériques présentées dans cette section sont donc toutes issues de la méthode à 2 grilles avec $\mathrm{N}$ $=2$. Le nombre de ddl de la grille fine est 2600 , celui de la grille grossière est égal à 726. L'exemple étudié concerne le flambage non linéaire d'un toit mince dont les caractéristiques géométriques et matérielles sont précisées sur la figure 5.

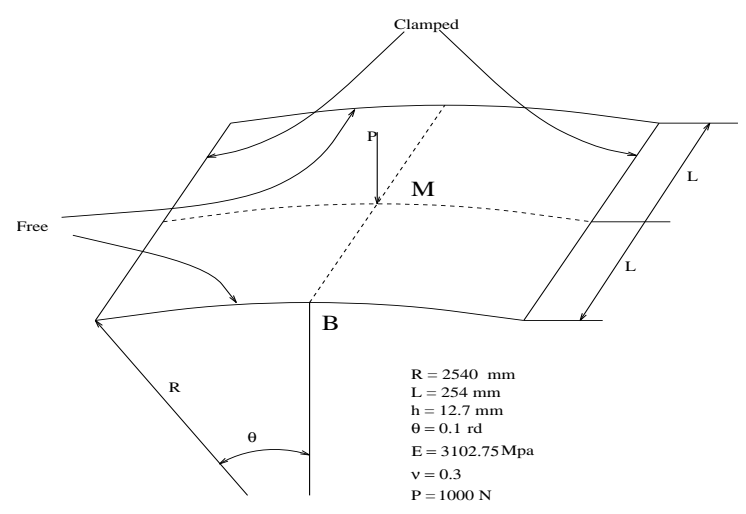

Figure 5. Description géométrique et matérielle du problème étudié

Nous présentons également sur la figure 6 la courbe « référence » qui est obtenue avec un solveur direct. Cette courbe référence représente l'évolution du déplacement vertical du point $\mathrm{M}$ (point d'application du chargement) en fonction de l'intensité du chargement (i.e. au paramètre de charge). L'ordre de troncature, $p$, de la MAN (nombre de problèmes linéaires à résoudre) est égal à 20. Le paramètre $\delta_{M A N}$, qui gouverne à 


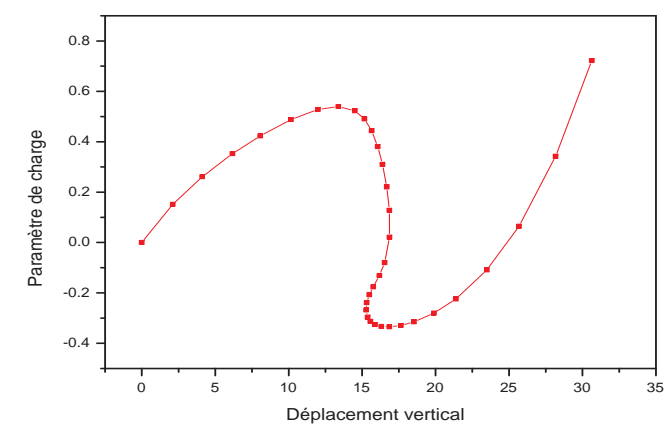

Figure 6. Courbe référence : déplacement vertical du point $M$ en fonction du paramètre de chargement

la fois la qualité de la solution MAN et le nombre de pas de calcul, est défini par la relation

$$
a_{\max }=\left[\delta_{M A N} \frac{\left\|u_{1}\right\|}{\left\|u_{p}\right\|}\right]^{\frac{1}{p-1}}
$$

où la notation $\|\bullet\|$ désigne la norme euclidienne d'un vecteur et $a_{\max }$ désigne la longueur maximum d'un pas de continuation MAN. La solution non linéaire est alors construite à partir du développement asymptotique :

$$
u=\sum_{i=1}^{p} a_{\max }^{i} u_{i}
$$

Pour obtenir cette courbe référence la valeur du paramètre $\delta_{M A N}$ a été fixée à $10^{-8}$.

Pour plus de précisions sur l'influence de ce paramètre sur la solution MAN, nous renvoyons le lecteur à la référence (Potier-Ferry et al., 2004).

\subsection{Influence de la précision du solveur linéaire sur la solution MAN}

Nous indiquons dans le tableau 3, le nombre de cycles multigrilles nécessaires à la convergence des problèmes linéaires [1] obtenus avec la MAN. Ce tableau montre que le nombre de cycles de la méthode à deux grilles nécessaire à la convergence dépend peu de l'ordre $i$. Pour diminuer ce nombre de cycles, on peut diminuer la précision demandée sur la résolution de chaque problème linéaire. A noter que pour chaque problème linéaire [1], le critère de convergence retenu est défini par l'expression suivante :

$$
\frac{\left\|\left[k_{t}\left(u_{0}\right)\right]\left\{u_{i}\right\}-f_{i}\right\|}{\left\|f_{i}\right\|}<\delta_{\text {multigrilles }}
$$




\begin{tabular}{|c|c|c|c|c|c|c|c|c|c|c|c|}
\hline & \multicolumn{10}{|c|}{ Nombre de cycles multigrilles } \\
\hline Ordre MAN & 1 & 3 & 5 & 7 & 9 & 11 & 13 & 15 & 17 & 19 & 20 \\
\hline$\delta_{\text {multigrilles }}=10^{-9}$ & 6 & 4 & 5 & 5 & 5 & 5 & 4 & 5 & 4 & 4 & 5 \\
\hline$\delta_{\text {multigrilles }}=10^{-8}$ & 6 & 4 & 4 & 4 & 4 & 4 & 5 & 4 & 4 & 4 & 4 \\
\hline$\delta_{\text {multigrilles }}=10^{-7}$ & 5 & 3 & 4 & 4 & 4 & 4 & 3 & 3 & 3 & 3 & 4 \\
\hline$\delta_{\text {multigrilles }}=10^{-6}$ & 4 & 3 & 3 & 3 & 3 & 3 & 3 & 3 & 3 & 3 & 3 \\
\hline$\delta_{\text {multigrilles }}=10^{-5}$ & 4 & 2 & 2 & 2 & 2 & 2 & 2 & 2 & 3 & 3 & 2 \\
\hline
\end{tabular}

Tableau 3. Influence de la précision du solveur multigrille sur le nombre de cycles de la méthode à deux grilles pour les différents ordres de la MAN. Cas d'un pas de calcul

Ainsi avec une qualité de solution $\delta_{\text {multigrilles }}=10^{-9}$, chaque problème linéaire demande 4 ou 5 cycles pour converger alors que dans le cas où $\delta_{\text {multigrilles }}=10^{-5}$, seulement 2 à 3 cycles sont nécessaires. Ce paramètre de précision sur chaque problème linéaire influence également la qualité du problème non linéaire. Sur la figure 7, nous représentons l'évolution du logarithme du résidu du problème non linéaire en fonction du paramètre de chargement et ceci pour plusieurs valeurs du paramètre

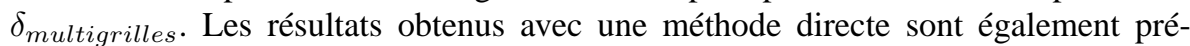
sentés. Cette figure montre que la qualité des solutions obtenue avec une méthode à deux grilles est équivalente à celle obtenue avec une méthode directe si les paramètres $\delta_{\text {multigrilles }}$ et $\delta_{M A N}$ sont égaux. Ce résultat a également été montré par (Galliet, 2000) et (El Mokhtari, 2002) lors du couplage de solveurs itératifs avec la MAN où une méthode de sous-structuration et un gradient conjugué préconditionné avaient été respectivement utilisés. Ces auteurs ont également montré que l'on pouvait diminuer le nombre d'itérations du solveur itératif en faisant évoluer la qualité de la solution des problèmes linéaires en fonction de l'ordre $i$. La figure 8 montre qu'il est inutile de calculer les termes d'ordre élevé avec une aussi grande précision que les premiers. Pour les quatre premiers ordres, le problème multigrille est résolu avec une précision $\left(\delta_{\text {multigrilles }}=10^{-8}\right)$. Pour les ordres supérieurs, la précision demandée est modifiée, avec par exemple $\delta_{\text {multigrilles }}=10^{-5}$ (stratégie 1 ) ou encore $\delta_{\text {multigrilles }}=10^{-6}$ (stratégie 2$)$.

\subsection{Cas de plusieurs pas de la méthode MAN}

La résolution du problème de la figure 5 nécessite de réaliser plusieurs pas MAN. Tous ces pas demandent approximativement le même nombre d'itérations. Il arrive cependant que pour certains pas, la méthode à deux grilles diverge. Pour l'exemple considéré, cette divergence survient pour une valeur du paramètre de chargement et du déplacement respectivement égale à $-0,3$ et 15 (voir figure 6). Afin de comprendre les causes de divergence de l'algorithme à deux grilles, plusieurs techniques de résolution ont été étudiées. Nous avons par exemple augmenté le niveau de factorisation de la matrice de préconditionnement lors des étapes de lissage. L'algorithme à deux grilles converge alors. Dans ce cas, la matrice de préconditionnement (lors de l'étape de lis- 


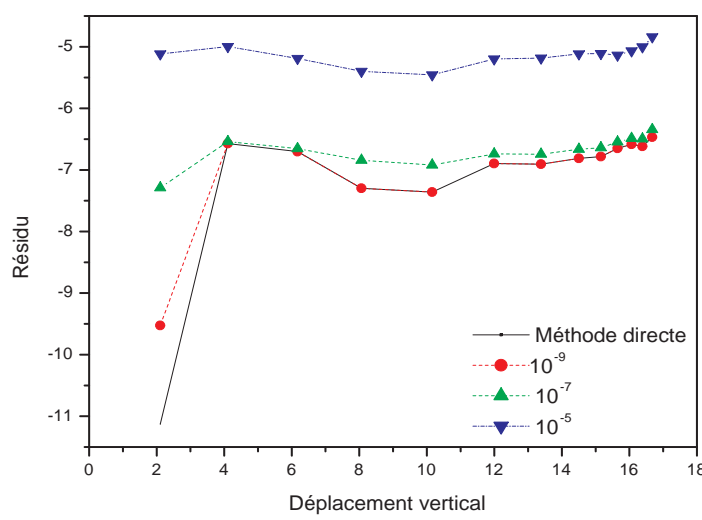

Figure 7. Evolution de la précision du calcul non linéaire de continuation, en fonction de la précision demandée pour la résolution du problème linéaire $\left(\delta_{\text {multigrilles }}\right.$ varie de $10^{-5}$ à $\left.10^{-9}\right) \cdot \delta_{M A N}=10^{-8}$. Problème du toit

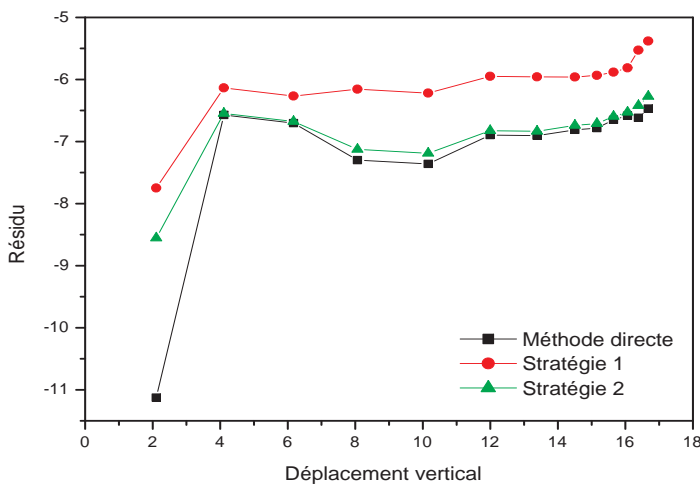

Figure 8. Précision du calcul non linéaire en fonction de la précision demandée pour

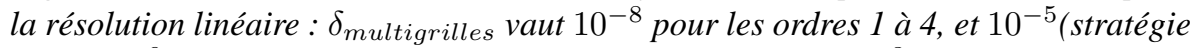
1) ou $10^{-6}$ (stratégie 2) pour les ordres suivants. $\delta_{M A N}=10^{-8}$. Problème du toit

sage) est alors très proche de la matrice réelle et cela conduit à résoudre les problèmes à l'aide d'une méthode directe. Nous avons également utilisé un gradient conjugué préconditionné (avec une factorisation incomplète de niveau 0) pour résoudre les pro- 
blèmes linéaires [1]. Ce dernier algorithme converge vers la solution avec la précision souhaitée sans difficulté.

La divergence de l'algorithme à deux grilles ne semble donc pas être due aux opérateurs définis sur la grille fine (matrice de préconditionnement ou matrice tangente de l'équation [1]). Cette divergence semble être liée aux opérateurs et aux quantités définis sur la grille grossière, par exemple la matrice de rigidité définie sur cette grille. L'étape 4 de l'algorithme à deux grilles présenté à la section 2.2 demande ainsi la résolution d'un problème linéaire qui est posé sur la grille grossière. L'opérateur permettant cette résolution sur la grille grossière est une matrice tangente qui, dans ce travail, est calculée à partir d'un vecteur déplacement défini sur la grille grossière et noté $U_{0}$. Ce dernier est évalué à partir de la restriction du vecteur déplacement défini sur la grille fine $u_{0}$, grâce à l'expression suivante :

$$
U_{0}=R u_{0}
$$

où $R$ est l'opérateur de restriction défini précédemment. Nous avons remarqué qu'une façon simple d'obtenir la convergence de la méthode à deux grilles était de modifier l'expression [8] de la manière suivante :

$$
U_{0}=\omega R u_{0}
$$

où $\omega$ est un scalaire. Ce paramètre joue le même rôle que les paramètres de sur ou sous-relaxation traditionnellement utilisés dans les méthodes itératives telles que la méthode de Jacobi. Tous les tests numériques réalisés dans cette étude ont montré que pour une valeur du paramètre $\omega$ proche de 1.0 (1.001 ou 1.05) la méthode à deux grilles converge pour tous les pas MAN. Ces valeurs du paramètre de sur-relaxation $\omega$ sont classiques avec les méthodes itératives. Ces tests numériques ont également montré que l'utilisation de l'expression [9] à la place de [8] ne modifie en aucune façon les performances de l'algorithme à deux grilles lorsque celui-ci converge avec l'expression [8]. La difficulté de cette méthode, comme pour toutes les méthodes de relaxation, est la dépendance du paramètre $\omega$ au problème étudié.

Finalement, la branche de la figure 6 a pu être calculée en 13 pas, avec les paramètres suivantes, $\delta_{\text {multigrilles }}=10^{-5}, \delta_{M A N}=10^{-8}$, le résidu du problème non linéaire restant toujours inférieur à $10^{-4.5}$ pour ce cas.

\section{Conclusions et perspectives}

Dans cette étude, nous avons couplé la méthode asymptotique à un solveur itératif de type méthode à 2 grilles. Les tests numériques montrent que dans le cas du flambage élastique non linéaire de coques minces nous retrouvons les performances de ces méthodes itératives : convergence rapide et indépendance du nombre de ddl de la grille fine. L'utilisation de cette méthode de résolution couplée à une méthode pas-àpas a cependant montré certaines difficultés de convergence. Cette difficulté a été levée en utilisant un pseudoparamètre de sur-relaxation. Les tests numériques montrent 
également que ces techniques numériques demandent pour chaque problème linéaire issu de la MAN le même nombre d'itérations pour obtenir la qualité souhaitée. Ce nombre d'itérations peut être diminué en utilisant des méthodes nécessitant plus de deux grilles, telles que les cycles $\mathrm{V}$ ou $\mathrm{W}$ ou encore les techniques de full-multigrid. Ces algorithmes sont actuellement à l'étude. Leur implémentation permettra d'envisager des exemples à très grand nombre d'inconnues et ainsi de mesurer le gain obtenu par le couplage MAN/multigrille par rapport à un couplage MAN/méthode directe.

\section{Bibliographie}

Abichou H., Zahrouni H., Potier-Ferry M., "Asymptotic numerical method for problems coupling several non-linearities", Computer Methods in Applied Mechanicsand Engineering, vol. 191, n 51-52, p. 5795-5810, 2002.

Azrar L., Boutyour E. M., Potier-Ferry M., "Non-linear forced vibration of plates by an asymptotic-numerical method", Journal of Sound and Vibrations, vol. 252, n 4, p. 657674, 2002.

Batoz J., Dhatt G., Modélisation des structures par éléments finis, Editions Hermès, Paris, 1990.

Braikat B., Damil N., Potier-Ferry M., « Méthodes asymptotiques numériques pour la plasticité », Revue européenne des éléments finis, vol. 6, p. 337-357, 1997.

Brandt A., Guide to multigrid developpment., W. Hackbusch and U. Trottenberg, Editors, Multigrid Methods, LNM, Vol. 960, p. 220-312, Springer-Verlag, Berlin, 1982.

Cadou J. M., Cochelin B., Damil N., Potier-Ferry M., "ANM for Stationary Navier-Stockes equations and with Petrov-Galerkin formulation", International Journal for Numerical Methods in Engineering, vol. 50, p. 825-845, 2001.

Descamps J., Cao H., Potier-Ferry M., "An asymptotic numerical method to solve large strain viscoplastic Problems", Computational plasticity, Fundamentals and Applications, C.I.M.N.E., vol. 1, p. 393-400, 1997.

Désidéri J., Modèles discrets et schémas itératifs, Editions Hermès, Paris, 1998.

Elhage-Hussein A., Damil N., Potier-Ferry M., "An asymtotic numerical algorithm for frictionless problems", Revue européenne des éléments finis, vol. 7, p. 119-130, 1998.

El Mokhtari R., Solveurs multigrilles et méthode de perturbation, Thèse de doctorat, Université de Metz, 2002.

Farhat C., Crivelli L., Roux F., "Extending substructure based iterative solvers to multiple load and repeated analysis", Computer Methods in Applied Mechanics and Enginnering, vol. 117, p. 195-209, 1994.

Fish J., Pan L., Belsky V., Gomaa S., "Unstructured Multigrid Method For Shells", International Journal for Numerical Methods in Engineering, vol. 39, p. 1181-1197, 1996.

Galliet I., Une version parallèle des méthodes asymptotiques-numériques. Application à des structures complexes à base d'élastomères, Thèse de doctorat, Université de Marseille II, 2000.

Joly P., Vidrascu M., Quelques méthodes classiques de résolution de systèmes linéaires, Collection didactique, INRIA, 1994. 
826 REMN - 16/2007. Modélisations numériques en mécanique

Potier-Ferry M., Cadou J. M., "Basic ANM algorithms for path following problems", Revue européenne des éléments finis, vol. 13, n 1-2, p. 9-32, 2004.

Rey C., "Une technique d'accélération de la résolution de problèmes d'élasticité non linéaire par décomposition de domaines", Comptes Rendus de l'Académie des sciences, Paris, vol. 322, n 8, p. 601-606, 1996. 